\title{
The temporal structure of urban soundscapes
}

\author{
D. Botteldooren*, B. De Coensel, T. De Muer \\ Acoustics Group, Department of Information Technology, Ghent University, \\ St. Pietersnieuwstraat 41, B-9000 Ghent, Belgium
}

Received 9 March 2004; received in revised form 11 July 2005; accepted 18 July 2005 Available online 6 September 2005

\begin{abstract}
The influence of noise on the quality of the urban living environment has traditionally been studied focusing on negative effects on man, such as noise annoyance and sleep disturbance. Recently a more holistic approach, including positive and negative aspects as well as non-residential functions of the urban environment, has gained renewed interest. The label "urban soundscape" is often used to refer to this approach. Research towards quantification of the acoustic descriptors of the urban soundscape is, however, still in an early stage. This paper draws on the analogy with music to propose an indicator for studying the temporal structure of the urban soundscape. The link to self-organized criticality of the underlying system is drawn. The influence on the new indicator of road traffic noise, an important soundscape disturber in urban areas, is analyzed in detail.

(C) 2005 Elsevier Ltd. All rights reserved.
\end{abstract}

\section{Introduction}

During the past few decades studies on the effect of noise on man have focused on physical and mental health, trying to relate it directly or indirectly to noise exposure level. In many situations the unwanted health effect, sleep disturbance, or annoyance can be related to one particular intruding sound. Soundscape research takes a more holistic approach. The urban acoustic environment is regarded as an aggregate of many sounds that can evoke specific emotions. The soundscape is seen as an integral part of the urban living environment. This way, the soundscape

\footnotetext{
${ }^{*}$ Corresponding author. Tel.: + 32 92649968; fax: + 3292649969.

E-mail address: dick.botteldooren@ugent.be (D. Botteldooren).
} 
is not studied in isolation, but is interwoven within the whole context of visual environment (landscape), feeling of safety, perceived air quality, etc. Mismatch between different components of the living environment, including soundscape, may be at least partly responsible for a negative evaluation of its quality.

Urban soundscapes emerge naturally as a result of the typical activities that take place in the public area. Over time, urban soundscapes have evolved. Today, in many cases road traffic noise dominates the soundscape, often implying impoverishment and dulling of the living environment. Therefore, soundscape design should be included in future urban planning and mobility planning. This requires the selection and use of a number of quality indicators for the acoustic field.

To describe the outdoor acoustic field, some indicators have become very commonly used. The $A$-weighted averaged sound level $L_{A \text { eq }}$ has traditionally been used as a primary indicator because it is easy to measure and to calculate and it correlates reasonably well with perceived loudness and specific annoyance. For non-specific, retrospective noise annoyance rating of the immediate vicinity of one's dwelling, night (and evening) seems to play an important role. Hence $L_{\mathrm{dn}}$ (or $\left.L_{\mathrm{den}}\right)$ is chosen as a suitable indicator for long-term assessment.

An overview of recent developments in the area of urban soundscape research and relevant indicators for the acoustic field can be found in Ref. [1]. Field investigation has identified a number of principal components in the subjective description of urban soundscapes [2-4]. Generally speaking, loudness-related cues come out as an important component, but a factor related to the spectral structure [5] and one related to the temporal structure also often emerge. Sound quality measures have been suggested for soundscape analyses [6,7] since they tend to capture loudness, spectral content and short time fluctuations in a way that is more closely related to subjective preference. Although often mentioned in relation to noise annoyance, studies on the influence of supra-second temporal structure have been very rare. Björk [8] has reported laboratory research on the relation between temporal structure and (specific) annoyance, conspicuousness, and startle. They concluded that $L_{A \text { eq }}$ is an appropriate indicator, at least for annoyance and for the sound stimuli used. However, in relation to the present study it should be mentioned that all stimuli were periodic and rather artificial in nature.

This paper presents an indicator for the temporal structure of urban soundscapes that is inspired by music research. Section 2 relates urban soundscapes to music and self-organized criticality (SOC). The latter is so common in natural processes (and natural soundscapes) that music can be thought of as an imitation of this particular temporal structure. Section 3 introduces an indicator and applies it to categorize a set of sounds. The new indicator is contrasted with classical indicators of environmental noise dynamics. Section 4 considers road traffic noise as an important determinant for urban soundscapes and analyses to what extent temporal structure of traffic flows can be a source of music-like temporal structure in the urban soundscape.

\section{Music, self-organized criticality and urban soundscapes}

When one thinks of music and temporal structure, the term rhythm almost naturally comes to mind. Rhythm has been a key aspect of music through all ages, in all continents. It can be described as the variation of the duration of sounds over time, or as the collection of all periodic events that constitute the sound. In Western music, rhythms are usually arranged with respect to a 
time signature. Different time-scales in music can be distinguished [9]. At the micro-level, music consists of sound particles, down to the threshold of audible perception. On a somewhat larger scale, music is composed of sound objects, basic units of musical structure, e.g. notes. At the meso level the time-scale can be described by the divisions of form, such as musical phrases. At the macro-level finally, the overall musical architecture or form is revealed, on a time-scale spanning minutes, hours or even days.

More in general, soundscapes are essentially manifestations of rhythmic systems, both in the sonic and subsonic realm [10]. Measures to describe the rhythm in music can therefore possibly provide good criteria for analyzing (urban) soundscapes. In natural and urban sounds, time structure at the micro-level - a few seconds and shorter - is typically associated to variations within one acoustic event. Time structure at the macro-level is caused by the succession of acoustic events. Also in the urban soundscape, the magnitude of loudness fluctuation or soundscape dynamics, is only slightly correlated to the temporal structure.

In a holistic approach, the temporal structure of music was studied by looking at the power spectrum of amplitude variations [11,12]. For this, the spectral density of the envelope of various pieces of music was calculated. In this type of spectrum, periodic events will be revealed as peaks, e.g. a loud note played about every $10 \mathrm{~s}$ will give a peak at $0.1 \mathrm{~Hz}$. Rhythmic structures on macro(low frequencies) as well as on micro-level (high frequencies) can be analyzed in this spectrum. The same was done for the time series of the instantaneous frequency, which can be seen as a simple model for pitch. It has been found that, on a log-log scale, the spectra for many of the musical genres considered were linear; moreover the slope always corresponded to $1 / f$ at the macro-level down to the length of the piece [11,12]. Fig. 1 shows a few examples of amplitude $\left(S_{p A}^{2}\right)$ and pitch $\left(S_{Z}^{2}\right)$ envelope spectra for different musical genres. In view of application of this technique for outdoor sound, the short-time $A$-weighted level time series of the acoustic signal can be used as the amplitude envelope, as it will be demonstrated in the next section.

In 1987, Bak et al. [13] introduced the notion of SOC to explain $1 / f$ noise. Although it is doubtful that their initial model actually succeeded in predicting $1 / f$ dynamics, SOC is now generally believed to be a source of linear $\log -\log$ behavior of complex systems $[14,15]$. So in creating music, man seems to imitate the temporal fluctuation of self-organized critical systems, which are quite common in the (natural) living environment. The observation that most listeners found artificial $1 / f$-type music more pleasing than artificial music showing a flatter or a steeper spectral slope [11] was also found to be correlated to characteristics of the chaotic dynamics observed in electroencephalograms of subjects listening to this music [16].

Based on the above observations, it must be concluded that the spectrum of loudness and pitch variations of a sound fragment, reveals important characteristics of this sound fragment. Moreover, it seems reasonable to assume that a straight spectrum in a log-log chart and in particular a $1 / f$ characteristic contributes to the pleasing character of the sound. By extension, this feature could be an interesting descriptor of urban soundscapes. In earlier work [17], it was shown that $1 / f$ spectral characteristics could indeed be found in the temporal pattern of amplitude and pitch in natural, urban, and rural soundscapes. Fig. 2 shows a few selected examples where this characteristic is quite obvious. The observation of $1 / f$ noise in these sounds becomes far less surprising when recognizing the complexity in the underlying systems as was shown in Ref. [17]. As examples we mention SOC that could arise under certain conditions in the passage of talking people or the passage of cars on a highway. Complex dynamics governing 

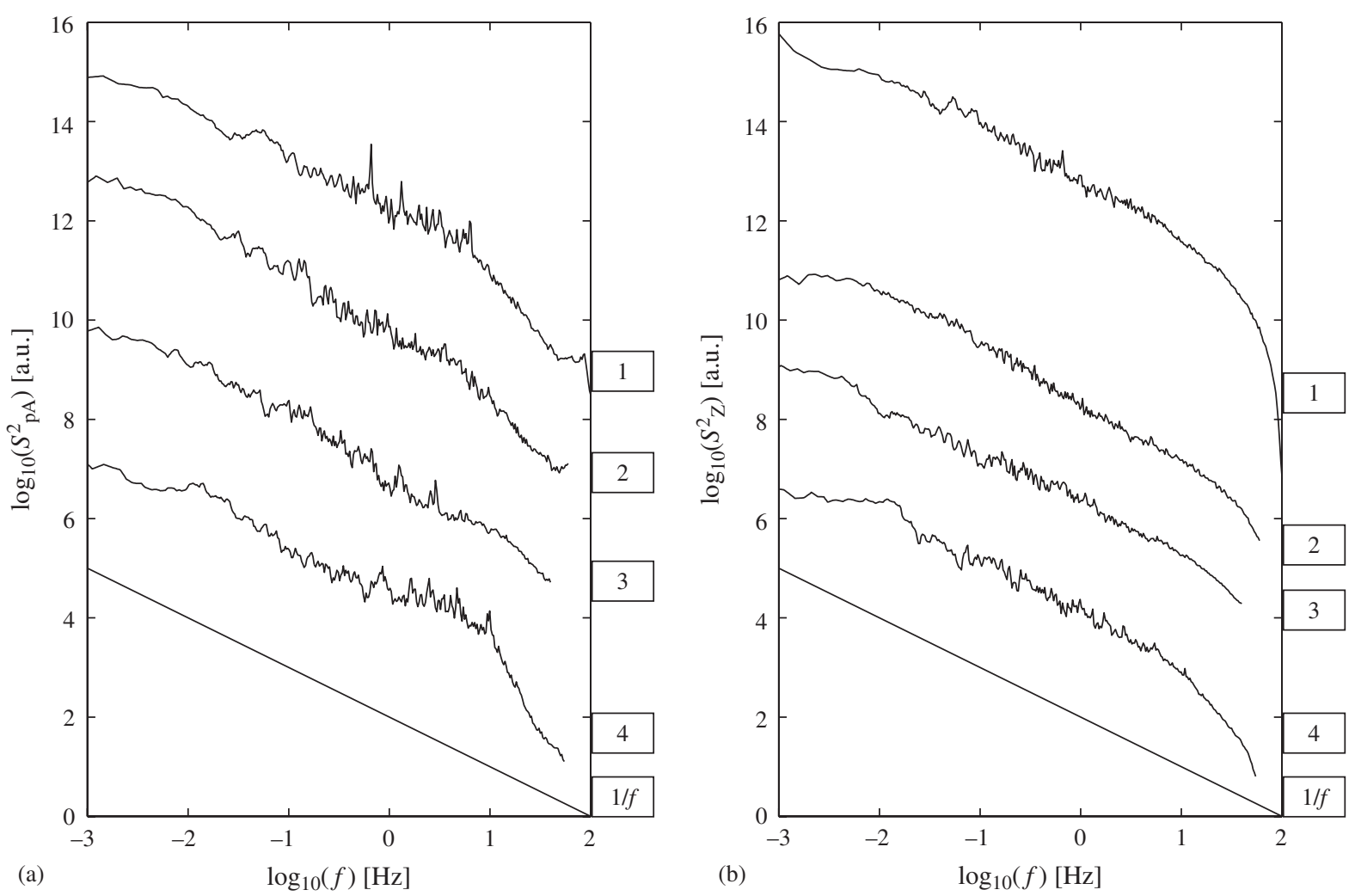

Fig. 1. Examples of (a) amplitude and (b) pitch spectra of music, compared to a $1 / f$ spectrum: (1) Brandenburg Concerto No. 1 by J.S. Bach; (2) Piano Concerto No. 2 by S. Rachmaninov; (3) Requiem by W.A. Mozart and (4) Four Seasons by A. Vivaldi.

natural sound include the chorus of birds singing or the sound of wind blowing in trees [17]. It is needless to say that many urban soundscapes show a much less appealing temporal pattern than those studied in this earlier work, mainly due to the dominating presence of road traffic noise.

The relationship between appealing temporal structure of an urban sound and impact indicators such as sleep disturbance or noise annoyance is not trivial. Interesting, music-like temporal structure may become quite disturbing or annoying when it intrudes unwantedly into ones living environment. It is even reasonable to assume that a more predictable, boring, and dull temporal structure is preferred in this case. Sound, presented as artificial music to a listening panel, was found to be labeled too predictable, boring, and dull if its amplitude and pitch spectrum had a slope steeper than $1 / f[11]$.

\section{Descriptors for the temporal structure of a soundscape}

\subsection{Descriptors for the temporal structure based on the spectrum}

A descriptor for the temporal structure of a soundscape is proposed, which measures the similarity of its spectrum of loudness (and pitch) fluctuations to those typical for music. Several 

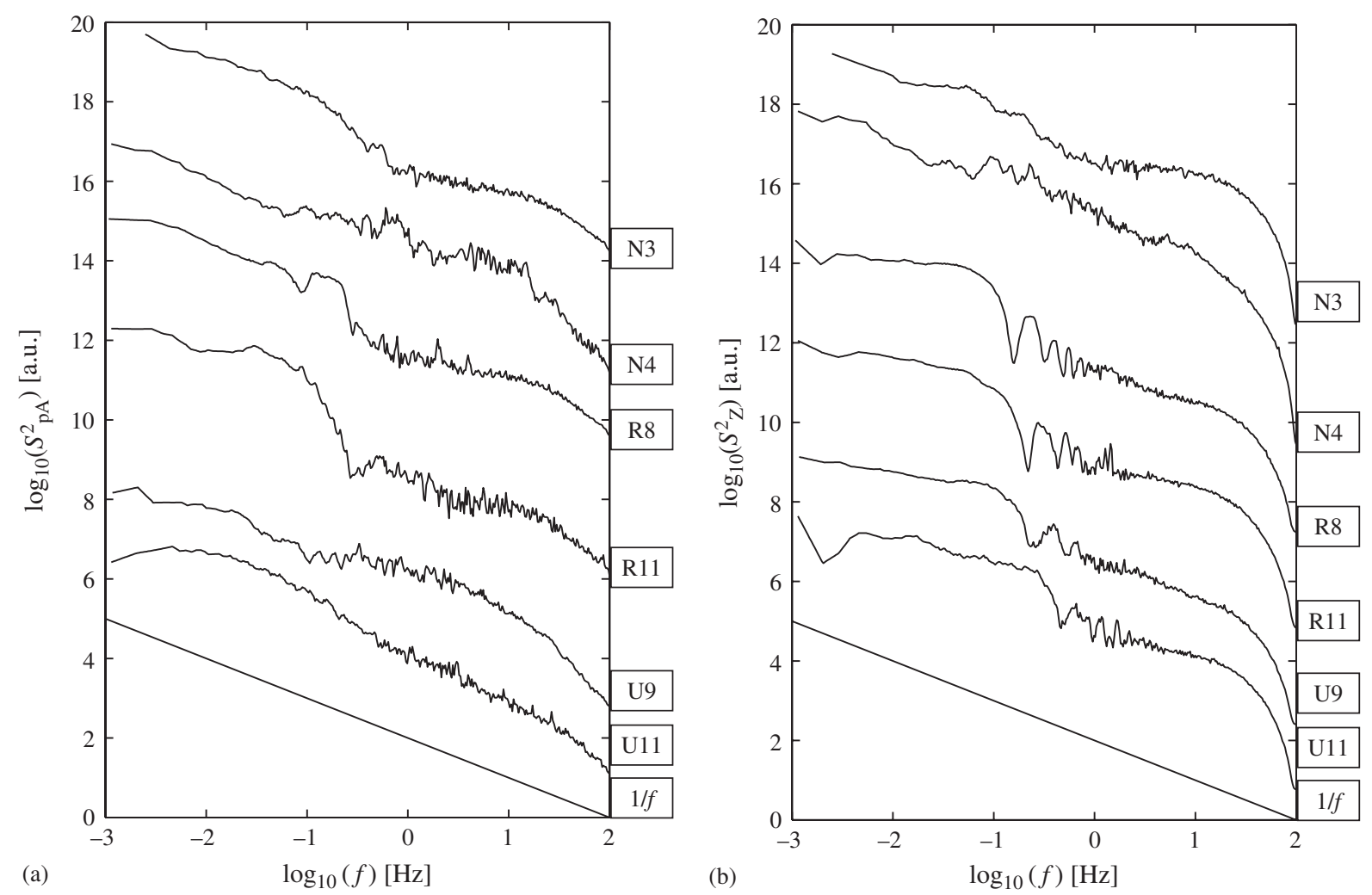

Fig. 2. Examples of (a) amplitude and (b) pitch spectra of natural (N3 \& N4), rural (R8 \& R11) and urban (U9 \& U11) soundscapes, compared to a $1 / f$ spectrum.

choices need to be made. The time interval of interest spans from a few hundred milliseconds to several minutes. It was already pointed out however that both for music and for urban soundscapes a critical point could be identified, between time structure at the micro- and macroscale, around a few seconds. For music, this critical point distinguished between the time structure determined by single notes and that determined by the musical phrase and longer length scales. Comparing the shorter length scale to urban noise seems less trivial because of this prevalent presence of rhythm in music. The frequency interval of interest is therefore split in $I_{1}=[0.002 \mathrm{~Hz}, 0.2 \mathrm{~Hz}], I_{2}=[0.2 \mathrm{~Hz}, 5 \mathrm{~Hz}]$ and $I_{3}=I_{1} \cup I_{2}$. The descriptor must further include not only the average slope of the spectrum but also a measure of its linearity (on a log-log scale). The latter is described by the quadratic deviation from the best-fitted straight line.

Careful investigation of the spectra in Fig. 1 shows that the so-called $1 / f$ slope found for music is not all that strict. It may be more appropriate to state that the amplitude and pitch spectrum of music has an approximate $1 / f$ or a $1 / f$-like behaviour. To quantify this vague statement, a fuzzy set containing all slopes $\alpha$ that are found in music is appropriate. The fuzzy set membership function is constructed on the basis of the probability distribution [18] of slopes, derived from the spectra of musical fragments, over the universe of slopes $U_{\alpha}$. To extract this probability 

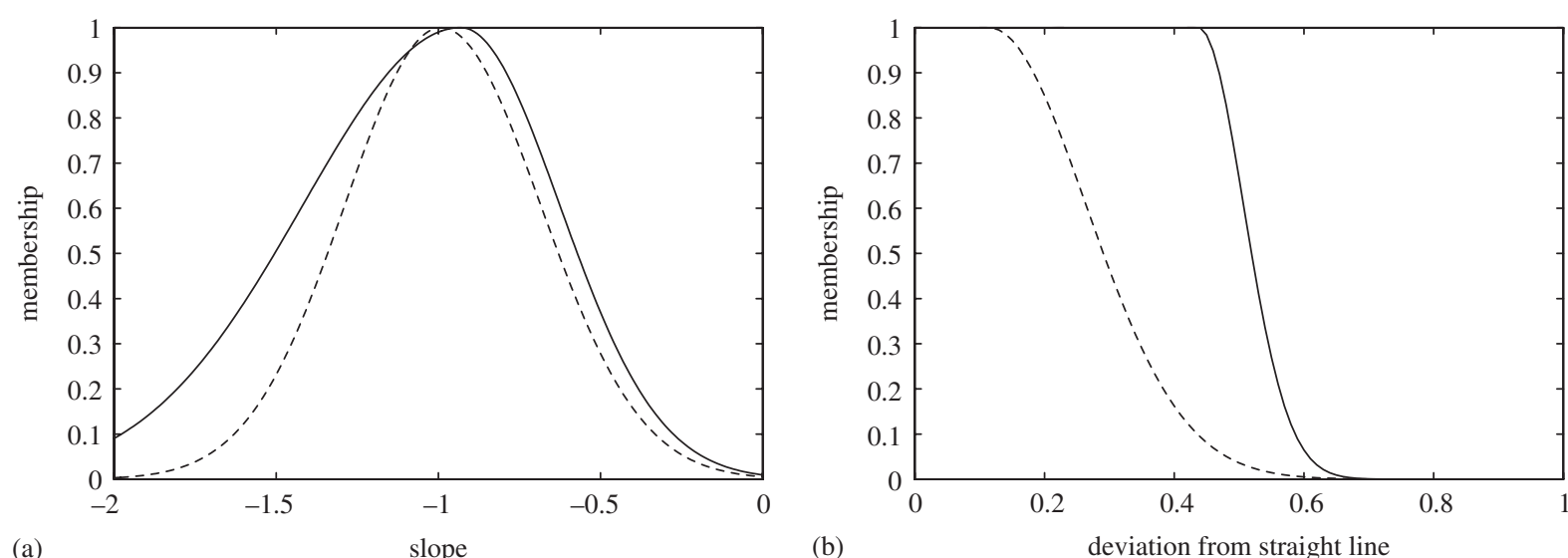

Fig. 3. Membership functions of the fuzzy sets describing (a) music-like slope of the amplitude spectrum and (b) musiclike deviation of the amplitude spectrum from a straight line. Both spectral intervals $I_{1}$ (solid lines) and $I_{2}$ (dashed lines) are considered.

distribution, 15 samples of music of different genres ( 3 pop, 3 jazz, 9 classical) are analyzed. Smoothened membership functions of music-like spectral slope, $S$, are shown in Fig. 3(a). Much in the same way, the deviation of the spectrum from a straight line must be approached. Smaller values of this deviation are more music-like thus leading to the inclusive fuzzy set membership functions, $D$, shown in Fig. 3(b). Both sets of membership functions are indexed by the spectral interval considered. Set membership degrees (values of the membership function) close to one mean perfect inclusion or a very music-like slope, $s$, or deviation, $d$. Membership degrees close to zero mean that the spectrum is very unlike that of music.

Evaluation of degree of music-likeness (ML) of the temporal structure of a soundscape is based on the measured slope in the spectrum of amplitude fluctuations, $s_{1}$ and $s_{2}$, and on the deviation from a straight line of this spectrum, $d_{1}$ and $d_{2}$. One of the following rules is used:

$\begin{array}{lll}\text { ML1 } & \text { IF } & s_{1} \in S_{1} \text { and } d_{1} \in D_{1} \\ & \text { THEN } & \text { temporal structure of sound is music-like } \\ \text { ML1\&2 } & \text { IF } & s_{1} \in S_{1} \text { and } d_{1} \in D_{1} \text { and } s_{2} \in S_{2} \text { and } d_{2} \in D_{2} \\ & \text { THEN } & \text { temporal structure of sound is music-like }\end{array}$

As an illustration of the use of these descriptors, Tables 1 and 2 contain resp., a verbal description of the most music-like and the least music-like soundscapes out of recordings at 45 randomly chosen locations (typical duration of a recording is $15 \mathrm{~min}$ ). Not surprisingly, the most music-like samples contain a variety of sounds from only weakly correlated sources. The least music-like ones are often dominated by a single source. In some cases this source is present most of the time (e.g. busy traffic) but in others the source produces only a small number of loud events per hour (e.g. train noise). Note also that the natural soundscapes that show a very music-like temporal pattern are not very quiet ones. Very quiet natural sites got rated worse because the constant background hum was too predictable in comparison to the temporal structure of music. 
Table 1

Selection based on ML1 of most music-like soundscapes out of 45

\begin{tabular}{llll}
\hline Label & Description & ML1 & ML1\&2 \\
\hline R8 & $\begin{array}{l}\text { Rural environment, sounds of birds, sometimes sounds from farm } \\
\text { animals and a few farming activities, no local traffic, some distant traffic } \\
\text { noise occasional commercial aircraft a high altitude } \\
\text { Traffic free shopping street in the center of Gent, talking people passing } \\
\text { sometimes stopping for a few seconds, occasional biker, some distant } \\
\text { murmur of car urban traffic, occasional (every 15 min) church bell }\end{array}$ & 1 & 0.35 \\
& $\begin{array}{l}\text { Rural environment, very similar to R8 } \\
\text { Remote rural environment at early morning, almost exclusively bird } \\
\text { R11 }\end{array}$ & 1 & 0.5 \\
Nounds, no man-made noise & 1 & 0.5 \\
N3 & $\begin{array}{l}\text { Rocky coast, waves (approximately 0.5 m height) braking, little wind, } \\
\text { occasional insect and one or two people silently passing by } \\
\text { Nature reserve at the edge of town, sounds of different species of birds } \\
\text { not particularly close, some wind in trees, occasionally talking people on } \\
\text { bike }\end{array}$ & 1 & 0.95 \\
U1 & $\begin{array}{l}\text { Urban park in residential area, distant traffic of different sorts (cars, } \\
\text { motorbikes, trains) with occasionally distinguishable events, wind in } \\
\text { trees, a few birds, a local car about every minute }\end{array}$ & 0.95 \\
U11 & & 0.05 \\
& & 0.7 \\
\hline
\end{tabular}

Table 2

Selection based on ML1 of least music-like soundscapes out of 45

\begin{tabular}{llll}
\hline Label & Description & ML1 & ML1\&2 \\
\hline U16 & $\begin{array}{l}\text { Urban street canyon in residential area, between } 400 \text { and } 600 \text { vehicles per } \\
\text { hour }\end{array}$ & 0.05 & 0 \\
U17 & $\begin{array}{l}\text { Urban street canyon in residential area, between } 100 \text { and } 200 \text { vehicles per } \\
\text { hour }\end{array}$ & 0.05 & 0 \\
U18 & $\begin{array}{l}\text { Urban street in residential area, between } 500 \text { and } 600 \text { vehicles per hour, } \\
\text { highway on flyover in the distance }\end{array}$ & 0.05 & 0 \\
R12 & $\begin{array}{l}\text { Rural area, a number of loud recreational events, one low flyover of a } \\
\text { military aircraft, in between sounds of birds and some distant road } \\
\text { traffic }\end{array}$ & 0.05 & 0 \\
U20 & $\begin{array}{l}\text { Urban road, two lane access road carrying between } 2000 \text { and } 2500 \\
\text { vehicles per hour }\end{array}$ & 0.05 & 0 \\
R14 & $\begin{array}{l}\text { Rural area close to railway, natural sounds and wind in trees are most } \\
\text { important noise sources between train passages }\end{array}$ & 0.05 & 0 \\
U2 & $\begin{array}{l}\text { Urban road with shopping facilities, slow car traffic and tram passing by } \\
\text { in groups (about 500 vehicles per hour), few talking people }\end{array}$ & 0.05 & 0 \\
U21 & $\begin{array}{l}\text { Urban road, two lane access road carrying between } 2000 \text { and } 2500 \\
\text { vehicles per hour } \\
\text { Busy highway in open area at a distance of about } 100 \mathrm{~m}\end{array}$ \\
U23 & $\begin{array}{l}\text { Urban street canyon in residential area, between } 200 \text { and } 300 \text { vehicles per } \\
\text { hour, some talking people passing }\end{array}$ & 0 & 0 \\
U19 & $\begin{array}{l}0 \\
\end{array}$
\end{tabular}




\subsection{Comparison to classical descriptors for dynamics}

The generally accepted feeling that fluctuating noise is more annoying than continuous noise, led to the construction of a number of indicators for sound exposure that include a measure of level fluctuation. The Noise Pollution Level [19] for example, $L_{\mathrm{NP}}$, is defined as $L_{\mathrm{NP}}=$ $L_{A \text { eq }}+\left(L_{A 10}-L_{A 90}\right)$ or similarly $L_{\mathrm{NP}}=L_{A \text { eq }}+2.56 \sigma$, where $\sigma$ is the standard deviation of the sound level.

For 31 soundscapes, the music-like temporal structure was obtained using the rules ML1 and ML1\&2. In Fig. 4 this result is compared to $L_{A 5}-L_{A 95}$ as a classical indicator of dynamics. This figure shows that there is some correlation between both descriptors (Pearson $r^{2}=0.25$ for ML1 and $r^{2}=0.07$ for ML1\&2). Some correlation is clearly expected as it was already mentioned that quiet environments disturbed by the occasional loud event have a low score on being music-like while at the same time $L_{A 5}-L_{A 95}$ will be high. The scattered points indicate that the new descriptor that is proposed probes a different dimension of the soundscape. To further illustrate how the spectral shape gives additional information, the sound-level distribution and amplitude spectrum of two very different sounds are shown in Fig. 5. Both sounds have very similar distributions, although shifted in amplitude, but their spectrum is quite different. Indeed the sound of the highway consists of a large number of short random events. The level fluctuation of rustling of wind in trees is governed by slow variations in wind velocity. Since these fluctuations in wind velocity are the result of a complex phenomenon, the spectrum of amplitude fluctuations corresponds better to a straight line for this second sound.

\subsection{Relation of descriptors for temporal structure to urban soundscape perception}

It is our opinion, the relationship between the proposed descriptor for urban soundscape temporal structure and music, and the proven effect of music and music-like noise on mental state and possibly on health, are sufficient to justify its introduction. Nevertheless, it would be interesting to find out how the temporal structure of the soundscape influences the evaluation by

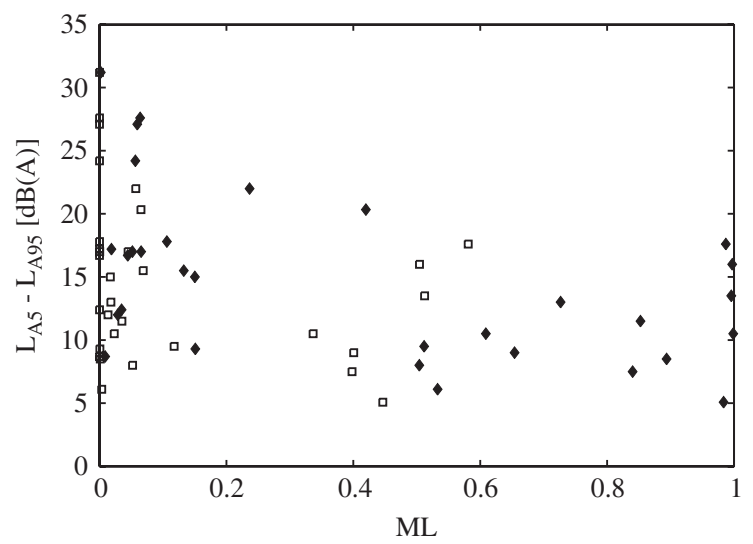

Fig. 4. Music-like temporal structure ML1 $(\bullet)$ and ML1\&2 $(\square)$ compared to $L_{A 5}-L_{A 95}$ as a classical measure of dynamics, illustrated for 31 soundscapes. 

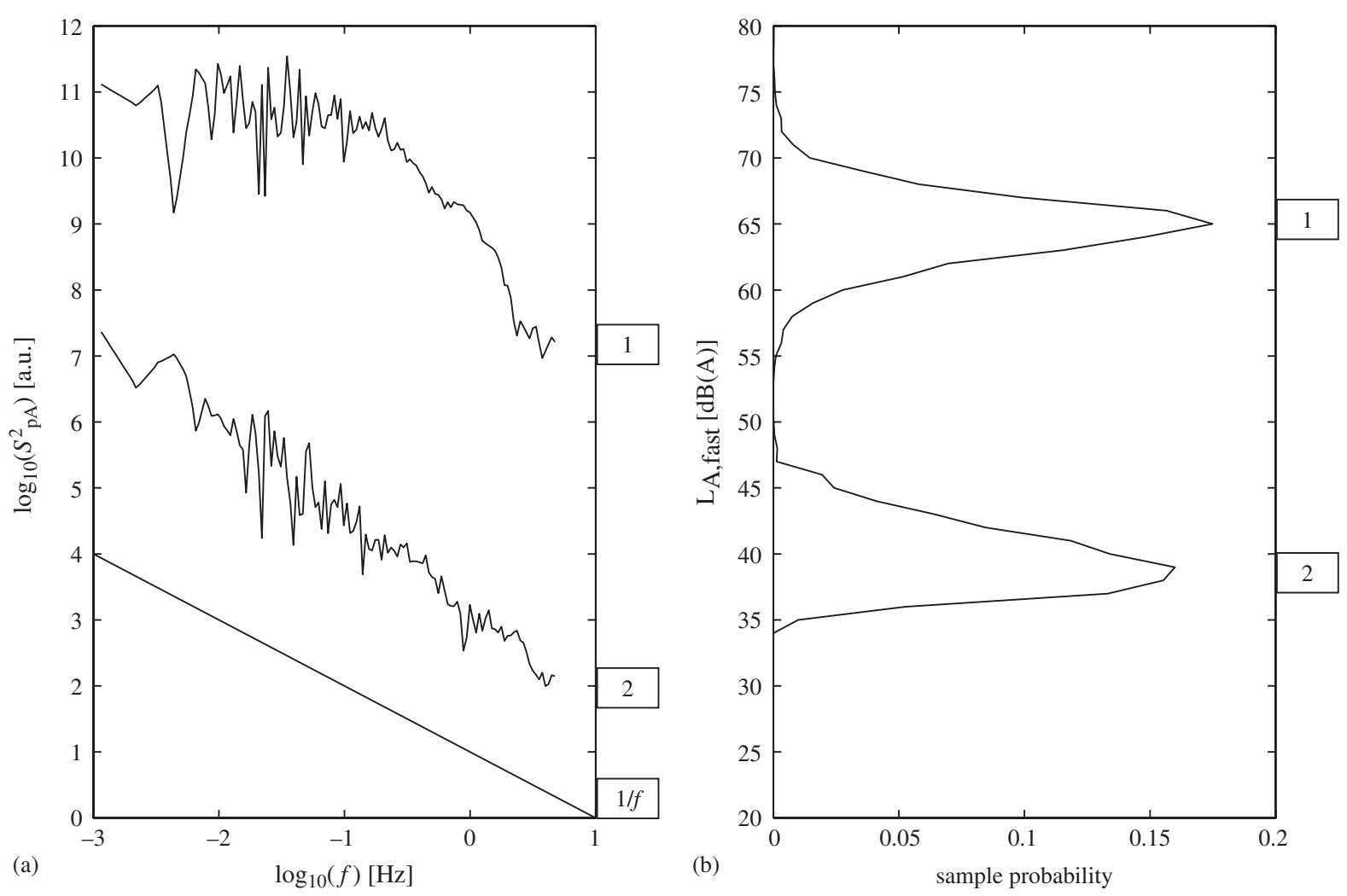

Fig. 5. Examples of (a) amplitude spectrum compared to a $1 / f$ spectrum and (b) sound-level distribution for two very different sounds: (1) the sound of a highway at short distance and (2) the rustling of wind in trees.

an accidental user of its urban context. A small-scale survey involving 100 subjects and 10 urban sites was set up as a pilot project. Since temporal structure involves time-scales of several minutes to a quarter of an hour, the questions had to have a retrospective character. Having people stand and listen to the urban sound for a quarter of an hour would just be too boring. Since the focus of this study is on soundscape characterization rather than on perception of sound, it was decided to separate the assessment based on the physical indicators from the interviews. The only two variables that were controlled were the season and the fact that it did not rain during any of the days of the experiment. The sample of passers-by was drawn at random, not controlling age or gender between the sites because there is an obvious natural bias.

Asking lay people about the temporal structure of a soundscape and its resemblance to music is impossible. The extent to which they can hear the music in urban sounds may depend strongly on their socio-cultural background and previous experience with more experimental types of music. Moreover, it could be expected that asking about music would trigger different foci, e.g. tonality, beat, etc. than the one envisaged in this work. To clarify the context, inspiration was found in the work of Voss and Clarke on music [11]. With respect to the slope in the spectrum of amplitude and pitch variations, it was found that a slope steeper than $1 / f$ resulted in sound that was labeled too boring and dull to be music, while a flatter slope resulted in a sound that was too chaotic and 


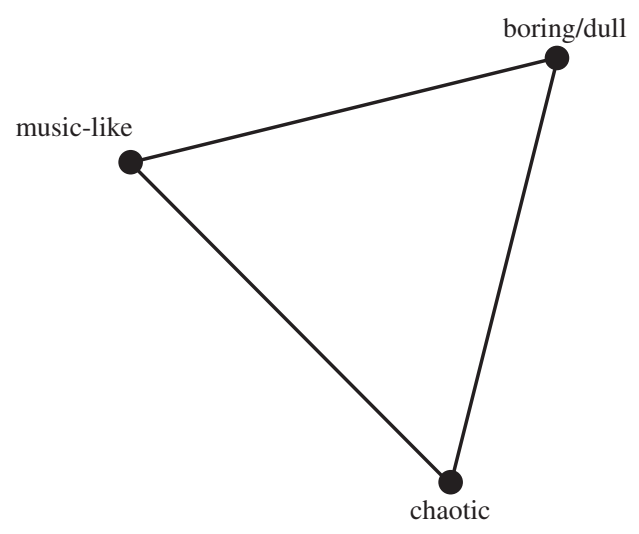

Fig. 6. Triangular answer scale used in the survey.

unpredictable to be music. Therefore it was decided to contrast music-like to boring/dull at one hand and to chaotic at the other hand. Because of the other connotations of music, it was decided not to place the label "music" exactly between chaotic and boring/dull as the work on temporal structure of music theoretically predicts. Instead the labels were put on a triangular scale (Fig. 6) and people were asked to put a mark in between the three points. Since the survey is conducted face-to-face, additional clarification of this rather difficult scale allows the average person to use it to express an opinion. The distance to each corner is measured for further analyses.

The relation between music-like temporal structure extracted from the spectral slope (ML1) and distance (average and 95\% confidence intervals) to the three points in the subjective scale, is given in Fig. $7(\mathrm{a}-\mathrm{c})$ for the 10 sites that were considered. The confidence intervals are relatively large, indicating the strong influence of other factors on the evaluation. Some personal factors can be expected to have an influence, but due to the limited size of the sample this could not be investigated. The clearest trend is seen for the distance from "chaotic". Soundscapes at sites where the objective parameter ML1 is high are subjectively rated further from chaotic. The subjective rating for music-like character shows the expected opposite trend: high ML1 corresponds to smaller distance to the subjective rating music-like. This trend is less pronounced. The subjective distance to boring/dull shows a trend that is opposite to what could be expected. Again inspired by the results obtained with music, a new variable was constructed that measures the shortest of the distances to boring/dull and chaotic. This variable was labeled "not like music" because both characteristics indicate that the temporal structure of the sound does not match a temporal structure that allows for this sound to be labeled "music". The correlation of this variable with ML1 is not much higher than the correlation of the variable "distance to chaotic", as can be seen in Fig. 7(d).

There are several methodological issues that may influence these results. Both the subjective perception and ML1 may be correlated to particular features of the sonic environment (e.g. the amount of road traffic) without being correlated to each other directly. Also, personal characteristics of typical passers-by may be different at different locations resulting in different average subjective evaluation not correlated directly to the noise itself. However, this small-scale study seems to confirm some of the observations made by trained acousticians. The unexpected 


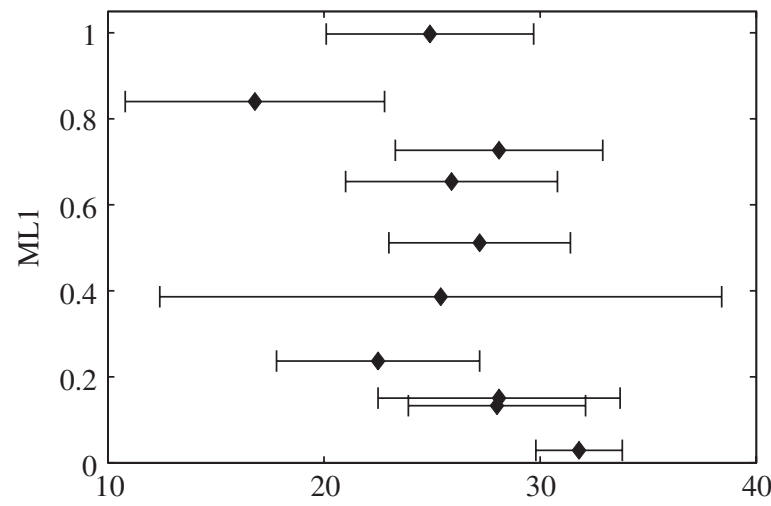

(a)

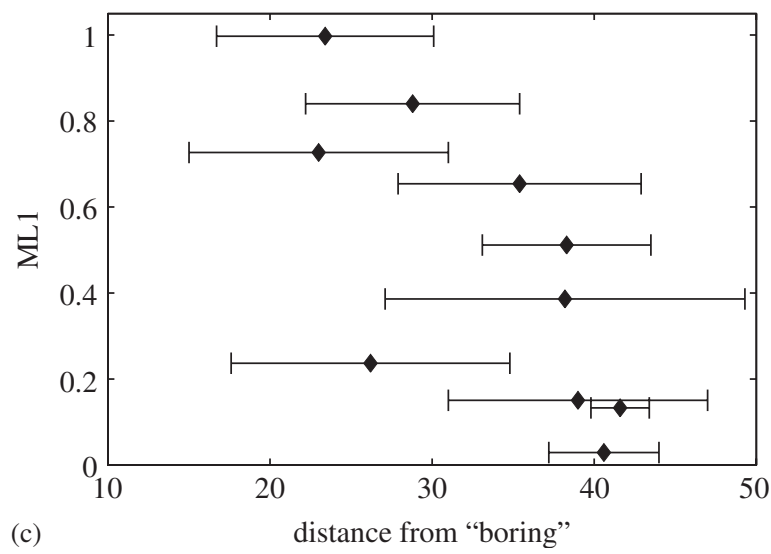

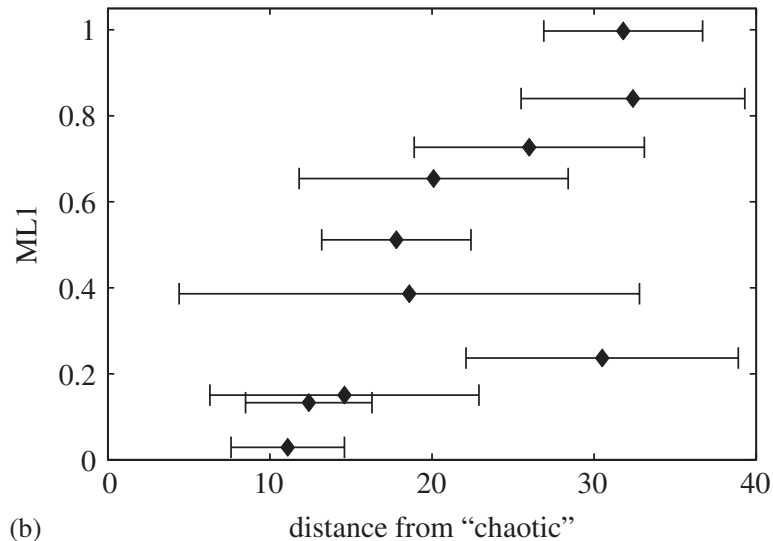

(b)

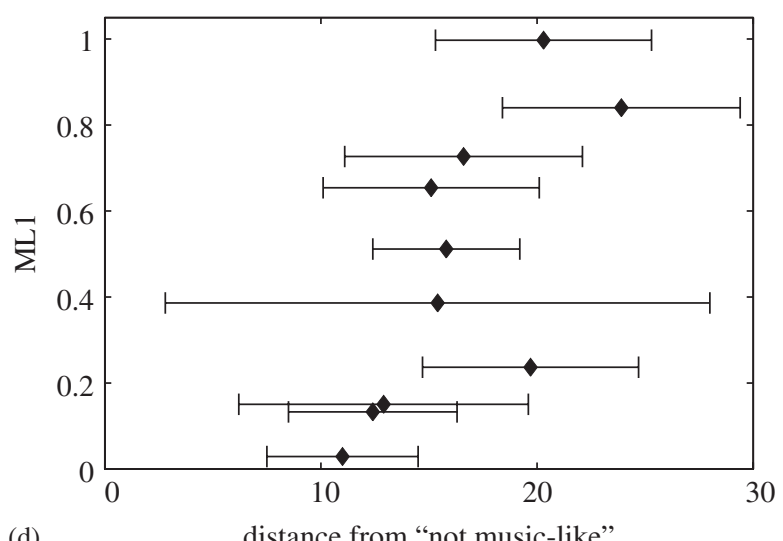

(d)

Fig. 7. Subjective evaluation of soundscapes in a triangle with the corners representing music-like, chaotic and boring/ dull, compared to the music-like temporal structure ML1. Averages and $95 \%$ confidence intervals are shown.

low value ML1 $=0.23$ for the fourth soundscape from below in Fig. 7 was also surprising for a trained observer. The broad confidence interval for the fifth soundscape from below in Fig. 7 seems to reflect the strongly changing character of the soundscape at this open urban square that was also noticed by the trained observer.

\section{Temporal structure of soundscapes dominated by road traffic noise}

In Table 2 it was observed that many of the soundscapes with non-music-like temporal structure were dominated by traffic noise. If it is impossible to remove road traffic noise from large areas of the urban structure, one may want to manage flows in such a way that they contribute as much as possible to the music-like temporal structure. It is not unreasonable to expect that there exists a traffic flow pattern that results in a $1 / f$ spectrum in the frequency interval $I_{1}$, since SOC has been observed in instantaneous traffic intensities [20-22]. Experimental studies involving traffic flows being very difficult to realize, a model for urban traffic 
noise based on microscopic traffic simulations was constructed and used to investigate temporal structure.

\subsection{Numerical model for urban traffic noise}

A numerical model for instantaneous traffic noise emission in urban area was developed and validated against experimental results [23]. The model is based on detailed micro-simulation of traffic flows [24]. This simulation considers every vehicle in the network as an independent entity interacting with the vehicles in its immediate vicinity. The influence of road saturation, traffic signals, crossings, speed limits, and vehicle fleet composition are automatically taken into account in the traffic flow calculation. Noise emission calculation distinguishes between vehicle categories and considers vehicle velocity [25]. Propagation is based on a polygonal beam tracer and includes multiple reflections and diffraction around vertical and horizontal edges. Details of this model can be found in Ref. [23].

\subsection{Temporal structure of traffic noise}

Before turning to simulated traffic flows, some insight is gained by analyzing analytical flows. First consider a flow of identical vehicles passing at the same speed along a straight road at randomly distributed instants. With these assumptions, the temporal structure of the sound pressure level only depends on the distance from the observer to the road axes and on vehicle speed. The spectrum can be obtained analytically by Fourier transformation of the Lorentz-curve; one finds,

$$
S_{p A}^{2}=\frac{W(n) \exp (-4 \pi f d / v)}{v^{2} d^{2}},
$$

where $v$ is the vehicle speed, $d$ is the distance between the observer and the road axis, and $W$ is the total sound power emitted by the traffic flow, which depends, among other things, on the traffic intensity $n$. Fig. 8(a) shows this spectrum for a few realistic combinations of distance, vehicle speed and traffic intensity, which are given in Table 3. In the inter-event time interval $I_{1}$, the spectrum is rather flat indicating chaotic and unpredictable temporal structure. At higher frequencies the decay is steep, an indication of predictability on this shorter time-scale. Random vehicle pass by instants is not a very realistic model unless vehicle intensity is so low that there is no interaction between the vehicles. Another extreme traffic model assumes that the distance between vehicles is constant. This makes the temporal structure periodic with a periodicity that reduces as traffic intensity increases. The spectrum (Fig. 8(b)) now peaks at a non-zero frequency corresponding to this periodicity. As $d / v$ increases this peak becomes sharper. Because of the $\log -\log$ plot, the peak is also more pronounced as its frequency increases. From the soundscape perspective this implies that in this second extreme traffic model also the temporal structure is far from music-like.

Let us now turn to more realistic traffic flows. Using the model described in the previous section, part of the city of Ghent is modeled in detail (Fig. 9). For each simulation, traffic is allowed to settle for $15 \mathrm{~min}$ before the 15 -min acoustic simulation starts. Sound levels, averaged over $0.5 \mathrm{~s}$, are calculated for a number of locations in this test area at a distance of $1 \mathrm{~m}$ from the 

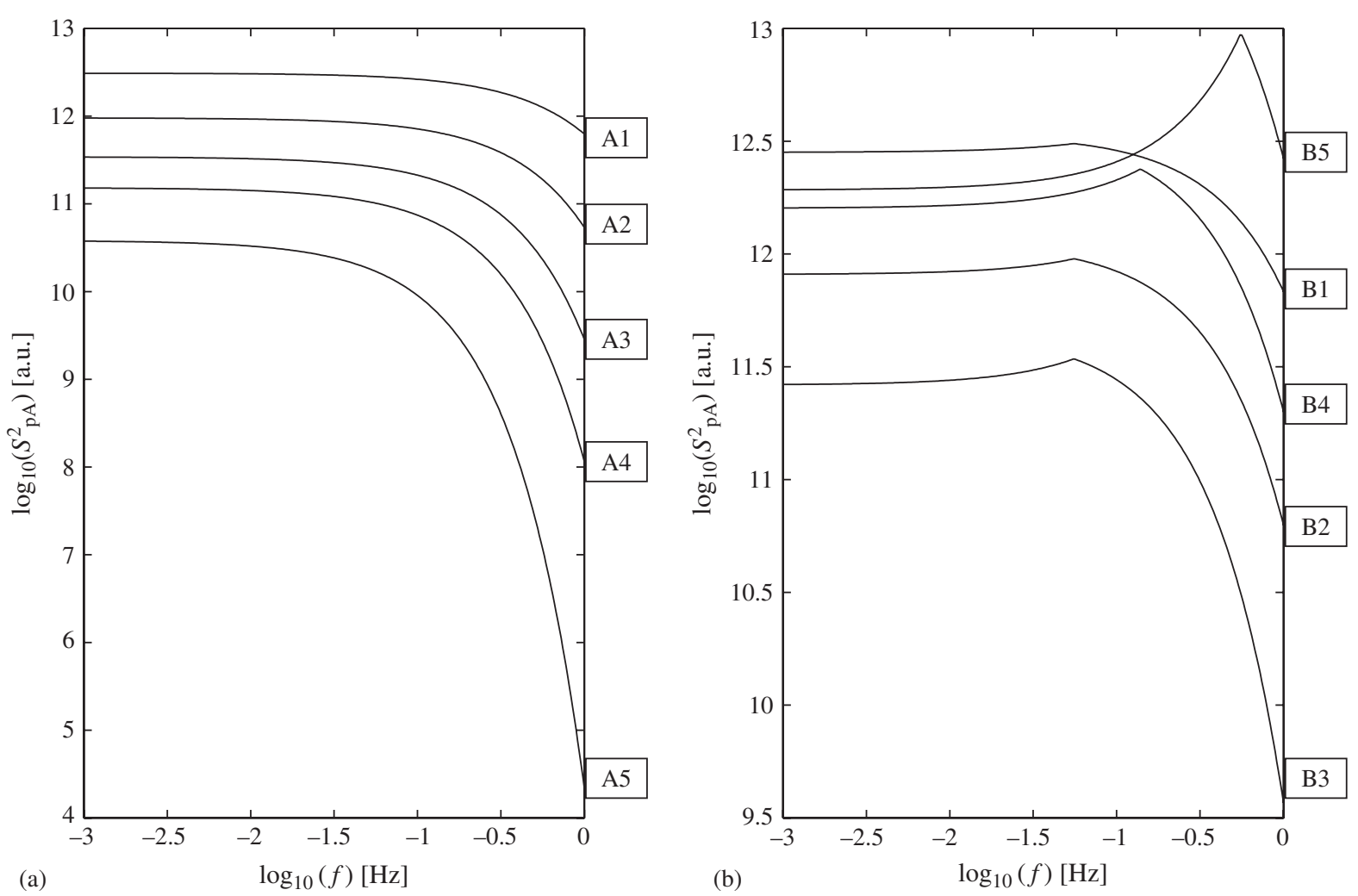

Fig. 8. Spectrum of amplitude fluctuations of the noise from an analytic traffic flow: (a) random vehicle instants and (b) equidistant vehicles. Parameter values can be found in Table 3.

Table 3

Parameter value combinations for the spectra shown in Fig. 8

\begin{tabular}{lrlc}
\hline Label & $d(\mathrm{~m})$ & $v(\mathrm{~km} / \mathrm{h})$ & $n(1 / \mathrm{h})$ \\
\hline A1 & 20 & 90 & 200 \\
A2 & 20 & 50 & 200 \\
A3 & 20 & 30 & 200 \\
A4 & 50 & 50 & 200 \\
A5 & 100 & 50 & 200 \\
B1 & & 90 & 200 \\
B2 & 20 & 50 & 200 \\
B3 & 20 & 30 & 200 \\
B4 & 20 & 50 & 500 \\
B5 & 20 & 50 & 2000 \\
\hline
\end{tabular}

façade. As a first example the spectra of the amplitude fluctuations and the sound-level distributions at 10 locations (one every $5 \mathrm{~m}$ parallel to the road axis) near measurement point 1 in the map are shown in Fig. 10. In the frequency interval $[0.01 \mathrm{~Hz}, 1 \mathrm{~Hz}]$, the spectrum resembles 


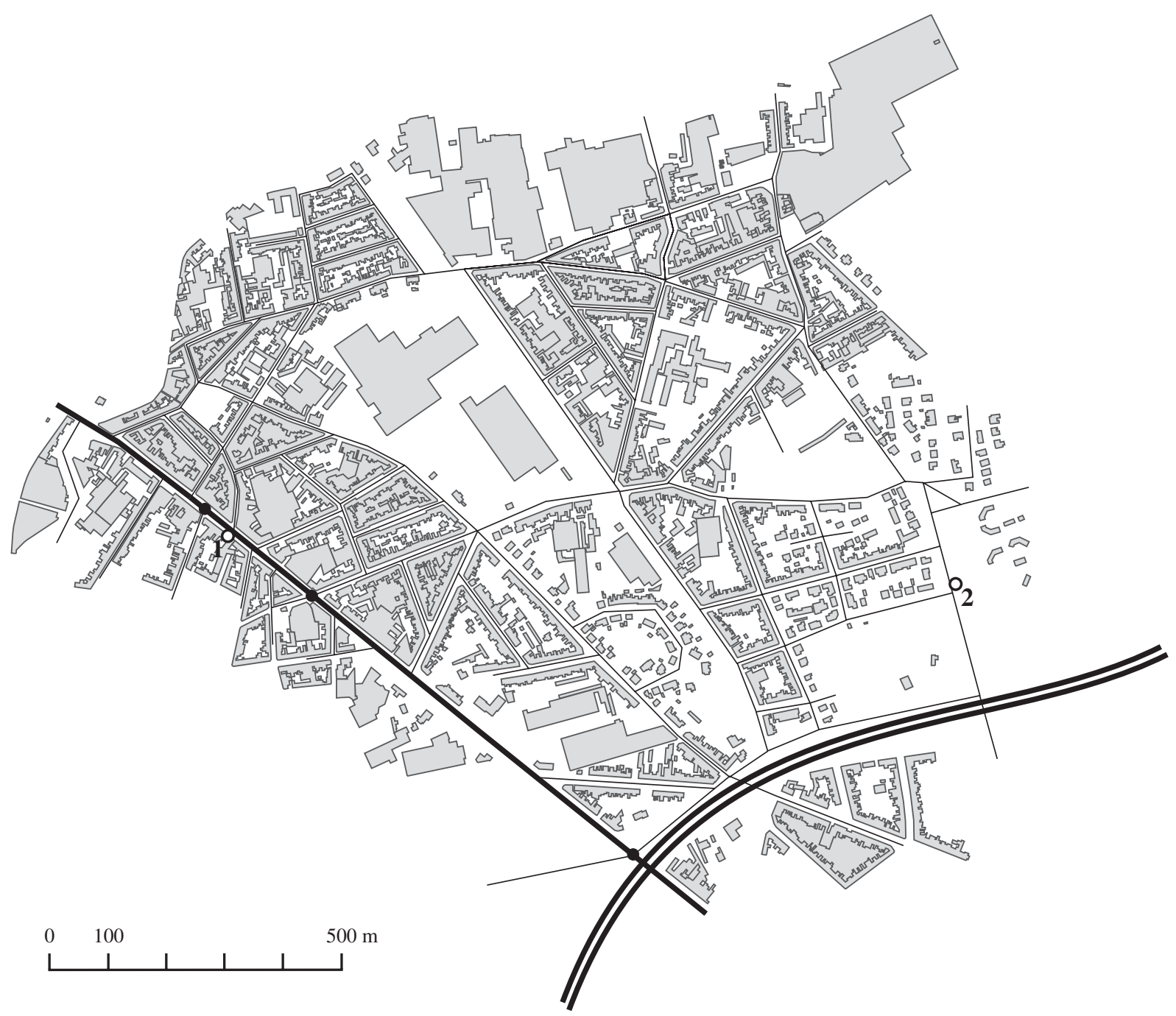

Fig. 9. Urban area used for simulating the impact of real traffic flow conditions on temporal structure of the soundscape. Closed dots on the main district road to the west indicate traffic lights; the measurement points are indicated by a circle.

more that of a complex system than the analytical approximations of Fig. 8 but the slope is not steep enough for this sound to show clear music-like dynamics. The peak around $0.01 \mathrm{~Hz}$ is caused by the periodicity of three sets of traffic lights in the vicinity of the observation area. If traffic demand is increased to $160 \%$, traffic is almost continuous during the green phase of the traffic lights. The spectral peak at $0.01 \mathrm{~Hz}$ gets more pronounced as well as a few side peaks, leading to a less music-like temporal structure (Fig. 11). To get a more complete picture of the effect of traffic intensity on the temporal structure of the soundscape, the traffic demand on the main access road that passes measurement point 1 is gradually increased from zero to $220 \%$ of today's rush hour 

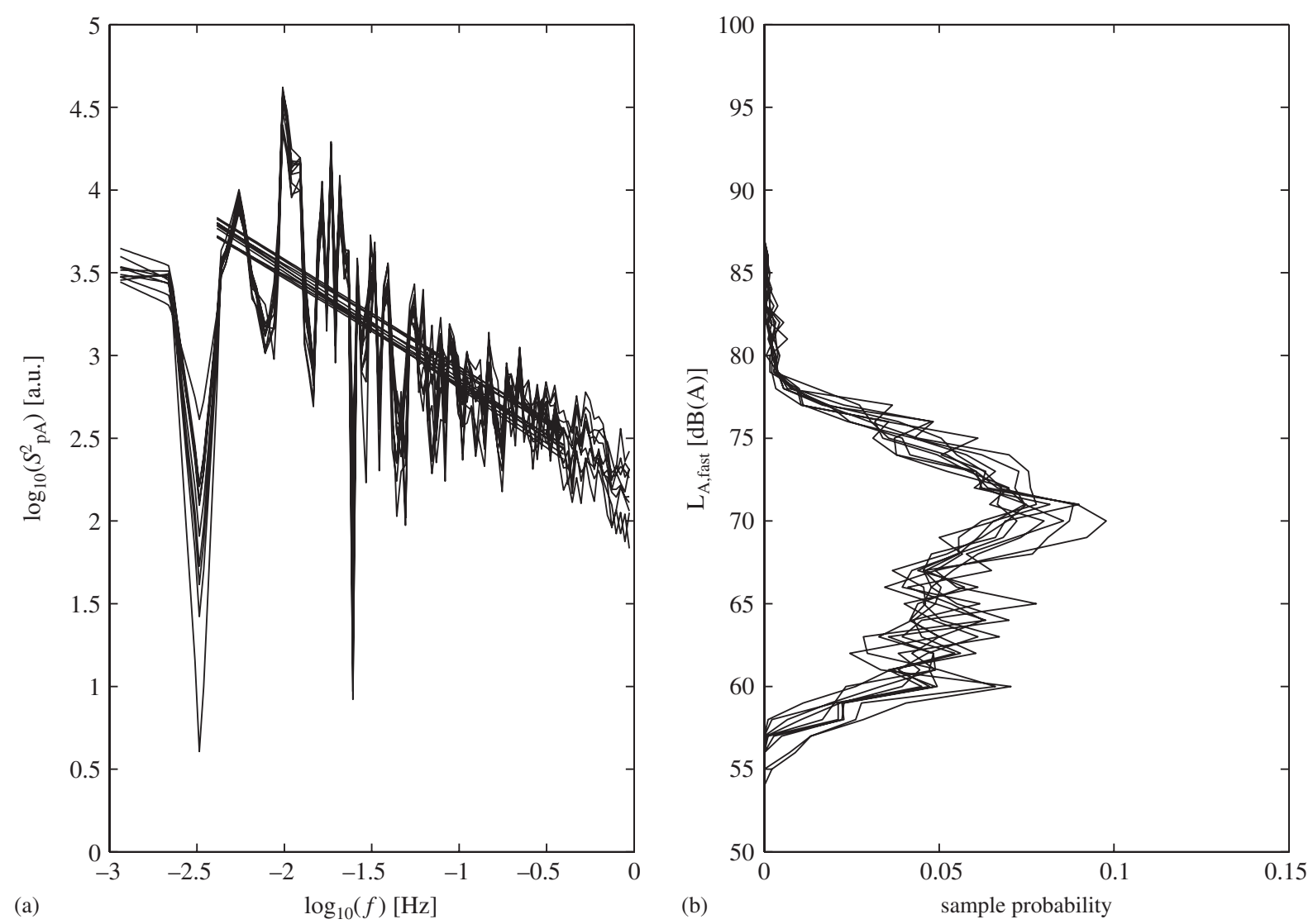

Fig. 10. (a) Amplitude spectrum and (b) sound-level distribution around point 1 in the map, with traffic at normal load during rush hour.

traffic in steps of 5\%. The indicator ML1 is calculated and averaged over 4 locations (one every $10 \mathrm{~m}$ ) near point 1 . Fig. 12(a) shows the increase of traffic intensity until the road totally saturates (including a complete jam at 190\%) and the evolution of ML1. Traffic noise shows a more musiclike temporal structure as traffic saturates, but probability seems to play an important role as indicated by the large spread in the points. Fig. 13(a) shows the steady decrease of $L_{A 5}-L_{A 95}$ with traffic demand. This decrease in dynamic continues after the road saturates and traffic intensity no longer increases.

As a second example, measurement point 2 is considered. Today, this is a quieter road through a less built-up area. Traffic dynamics are hardly influenced by traffic lights. Random generation of traffic at local nodes on the other hand plays a much more important role. Fig. 12(b) shows the mild saturation of traffic and the evolution of ML1. Traffic noise turns out to be much too random to be labeled music-like in this case. The small peak in ML1 at a traffic demand that is $200 \%$ of today's value may correspond to an onset of SOC. It corresponds to a slight increase in number of cars, corresponding to the general notion that the throughput is optimal at SOC conditions. Fig. 13(b) shows how the $L_{A \text { eq }}$ saturates more quickly than the traffic intensity as a 

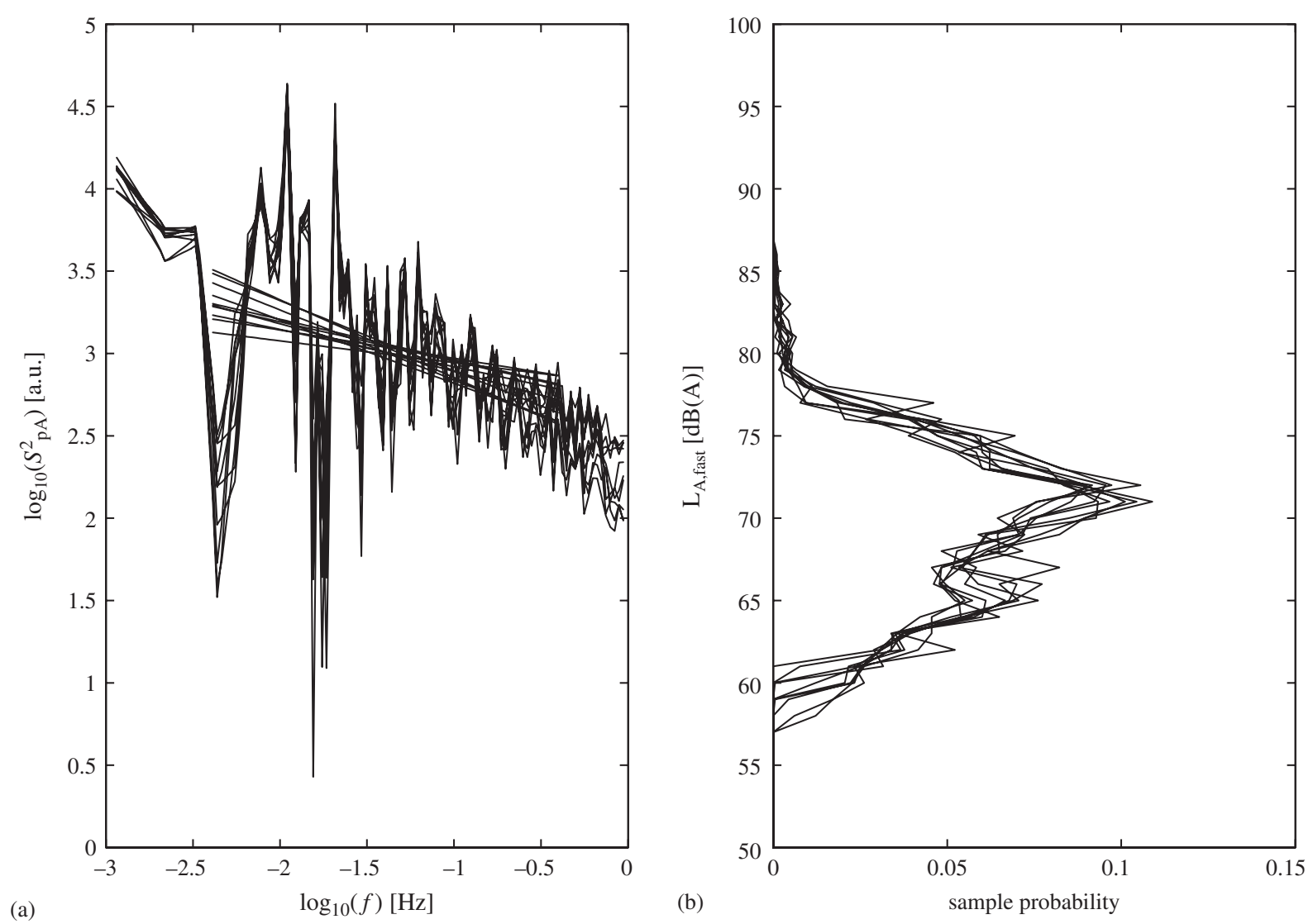

Fig. 11. (a) Amplitude spectrum and (b) sound-level distribution around point 1 in the map, with traffic at $160 \%$ of normal load during rush hour.
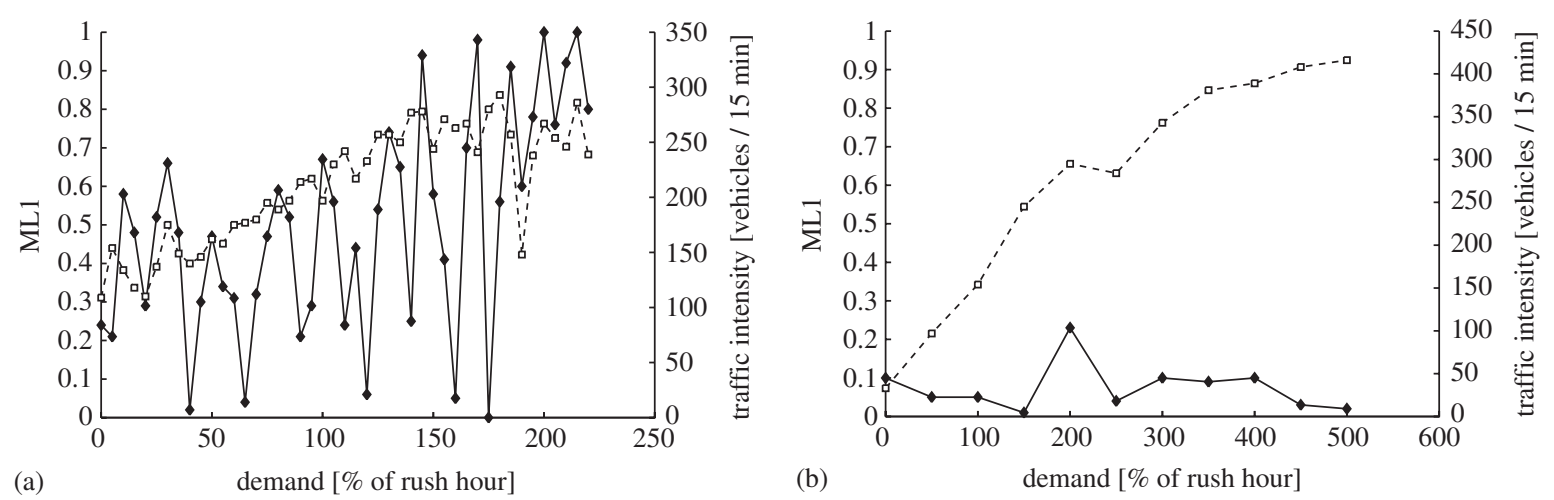

Fig. 12. Average music-like temporal structure ML1 of the traffic noise ( $\bullet$ ) and traffic intensity on one lane ( $\square$ ): (a) near location 1 as a function of traffic demand (in percentage of rush hour traffic) on the main access road and (b) near location 2 as a function of traffic demand on this local road. 

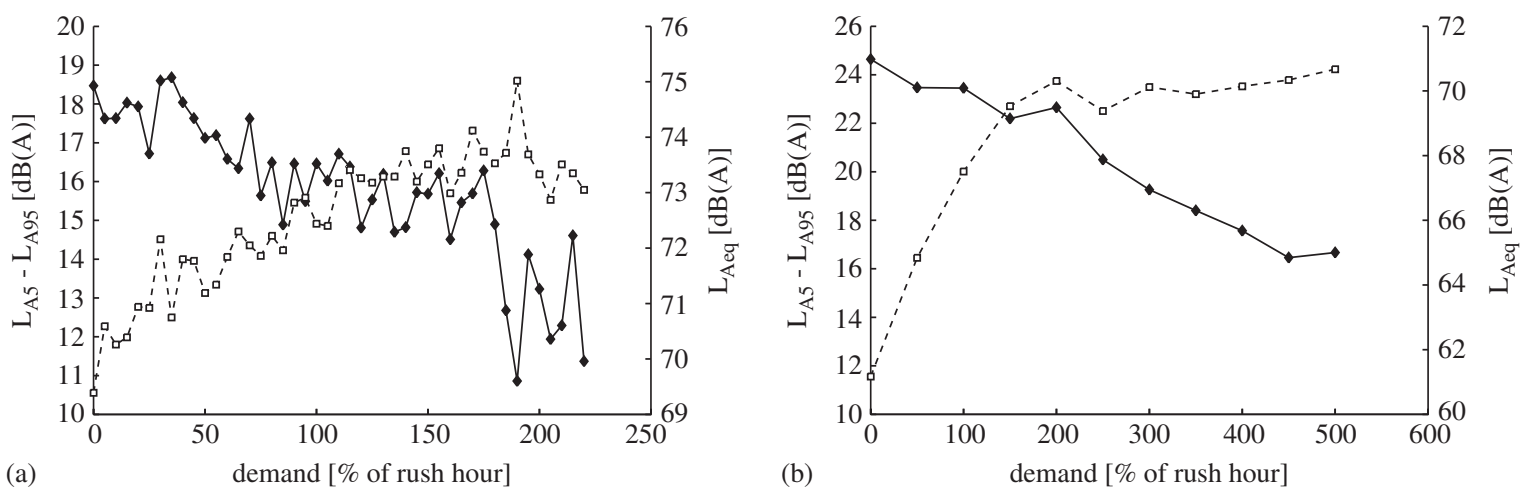

Fig. 13. $L_{A 5}-L_{A 95}(\diamond)$ and $L_{A \text { eq }}(\square)$ : (a) near location 1 as a function of traffic demand (in percentage of rush hour traffic) on the main access road and (b) near location 2 as a function of traffic demand on this local road.

function of traffic demand due to the reduced vehicle speed. Also, $L_{A 5}-L_{A 95}$ steadily decreases with traffic demand indicating the filling up of quiet intervals.

\section{Conclusions}

The temporal structure of an urban soundscape can accurately be described by looking not only at the dynamics in terms of differences in statistical noise levels, but also at the spectrum of amplitude (and pitch) fluctuations. Since it was noticed that particular spectral features that relate to self-organized criticality (SOC) are present in most types of music (so-called $1 / f$ noise) it is enlightening to look in particular for this feature in the temporal structure of urban soundscapes. By combining the requirement that the spectrum must show a straight line on a log-log scale and that this straight line must have a $1 / f$ slope for the temporal structure to be music-like, a fuzzy indicator for music-likeness (ML) was constructed. Environmental sound recordings were tested for this temporal structure and indeed it was found in several natural and urban environments. At the same time soundscapes with far from music-like temporal structure are also quite common. For this latter group, two situations occur: either the temporal structure is too predictable or it is too chaotic. In general it can also be observed that music-like temporal structure is much more rare in soundscapes dominated by a single source.

A small-scale survey indicates that the ML characteristic does not correlate well with the feeling that the environmental noise sounds like music, mainly because few subjects could imagine urban noise to be music. On the contrary, there seems to be a clearer relation to the soundscape being neither chaotic nor boring.

Traffic noise is an important contributor to many urban soundscapes. Using micro-simulation of traffic flow, it has been shown that music-like dynamics can emerge in traffic noise on the supra-event time-scale. SOC that emerges in the underlying traffic system is responsible for this behavior. Unfortunately this is not a very common situation in urban traffic noise. For free flow, traffic seems often to be too random or too structured. Randomness is caused by local generation 
of traffic. Traffic management in general, and traffic lights in particular, tend to regulate flow in a more deterministic manner.

The indicator presented in this paper sheds new light on how urban soundscape quality might be assessed in an objective way. By using the analogy with music, the indicator follows more closely the original ideas behind urban soundscape research. Based on this, we argue that the proposed indicator is a good candidate for describing and categorizing soundscape temporal structure, and can be used in addition to loudness and spectral-quality indicators (sharpness, roughness). There is an obvious need for further analyses of the relation between this objective indicator and more subjective evaluations of the quality of urban sound by an active participant. Dedicated research involving psycho-acoustic lab research as well as field investigation should be started in this field.

\section{References}

[1] P. Lercher, B. Schulte-Fortkamp, The relevance of soundscape research to the assessment of noise annoyance at the community level, in: Proceedings of the Eighth International Congress on Noise as a Public Health Problem, Rotterdam, The Netherlands, 2003, pp. 225-231.

[2] J. Kang, M. Zhang, Semantic differential analysis on the soundscape of urban open public areas, in: Proceedings of the First Pan-American/Iberian Meeting on Acoustics, Cancun, Mexico, 2002.

[3] B. Berglund, M. Nilsson, An attempt to capture the perceived soundscape, in: Proceedings of the International Symposium on Noise Pollution and Health (NOPHER), Cambridge, UK, 2001.

[4] S. Viollon, C. Lavandier, Multidimensional assessment of the acoustic quality of urban environments, in: Proceedings of Internoise (CDROM), Nice, France, 2000.

[5] B. Berglund, P. Hassmén, A. Preis, Annoyance and spectral contrast are cues for similarity and preference of sounds, Journal of Sound and Vibration 250 (1) (2002) 53-64.

[6] K. Genuit, The use of psychoacoustic parameters combined with $A$-weighted SPL in noise description, in: Proceedings of Internoise, Fort Lauderdale, Florida, USA, 1999, pp. 1887-1892.

[7] K. Genuit, Soundscape design — acoustical challenge, in: Proceedings of Forum Acusticum, Sevilla, Spain, 2002.

[8] E.A. Björk, Effects of inter-stimulus interval and duration of sound elements on annoyance, Acta Acustica United with Acustica 88 (1) (2002) 104-109.

[9] C. Roads, Microsound, The MIT Press, Cambridge, MA, 2002.

[10] J. Winkler, Rhythmicity, in: H. Järviluoma, G. Wagstaff (Eds.), Soundscape Studies and Methods, Finnish Society for Ethnomusicology, Helsinki, 2002, pp. 133-142.

[11] R. Voss, J. Clarke, " $1 / f$ noise" in music: music from $1 / f$ noise, Journal of the Acoustical Society of America 63 (1) (1978) 258-263.

[12] R. Voss, J. Clarke, $1 / f$ noise in music and speech, Nature 258 (1975) 317-318.

[13] P. Bak, C. Tang, K. Wiesenfeld, Self-organised criticality: an explanation of $1 / f$ noise, Physical Review Letters 59 (1987) 381-384.

[14] J. Davidsen, H.G. Schuster, $1 / f^{\alpha}$ noise from self-organised critical models with uniform driving, Physical Review $E$ 62 (5) (2000) 6111-6115.

[15] P. De Los Rios, Y. Zhang, Universal $1 / f$ noise from dissipative self-organised criticality models, Physical Review Letters 82 (3) (1999) 472-475.

[16] J. Jeong, M.K. Joung, S.Y. Kim, Quantification of emotion by nonlinear analysis of the chaotic dynamics of electroencephalograms during perception of $1 / f$ music, Biological Cybernetics 78 (1998) 217-225.

[17] B. De Coensel, D. Botteldooren, T. De Muer, $1 / f$ noise in rural and urban soundscapes, Acta Acustica United with Acustica 89 (2) (2003) 287-295.

[18] L.A. Zadeh, Fuzzy sets as a basis for a theory of possibility, Fuzzy Sets and Systems 1 (1978) 3-28. 
[19] M.J. Crocker, Rating measures, descriptors, criteria and procedures for determining human response to noise, in: M.J. Crocker (Ed.), Encyclopedia of Acoustics, vol. 2, Wiley, New York, 1997, pp. 943-965.

[20] M.Y. Choi, H.Y. Lee, Traffic flow and $1 / f$ fluctuations, Physical Review E 52 (6) (1995) 5979-5984.

[21] X. Zhang, G. Hu, $1 / f$ noise in a two-lane highway traffic model, Physical Review E 52 (5) (1995) 4664-4668.

[22] D. Chowdhury, A. Schadschneider, Self-organisation of traffic jams in cities: effects of stochastic dynamics and signal periods, Physical Review E 59 (2) (1999) R1311-R1314.

[23] B. De Coensel, T. De Muer, I. Yperman, D. Botteldooren, The influence of traffic flow dynamics on urban soundscapes, Applied Acoustics 66 (2) (2005) 175-194.

[24] http://www.paramics-online.com, paramics is being developed by Quadstone Ltd.

[25] H.G. Jonasson, S. Storeheier, Nord 2000: new Nordic prediction method for road traffic noise, Technical Report 2001:10, SP Swedish National Testing and Research Institute, 2001. 